\title{
Sunlight-induced Basal Cell Carcinoma Tumor Cells and Ultraviolet-B-irradiated Psoriatic Plaques Express Fas Ligand (CD95L)
}

\author{
Christina Gutierrez-Steil,, ${ }^{\star}$ Tamara Wrone-Smith, ${ }^{\star}$ Xiaoming Sun, ${ }^{\star}$ James G. Krueger, ${ }^{\ddagger}$ Todd Coven, ${ }^{\ddagger}$ and Brian J. Nickoloff* \\ *Department of Pathology and Skin Cancer Research Laboratories, Cardinal Bernardin Cancer Center, Loyola University of Chicago, \\ Maywood, Illinois 60153; and ${ }^{\ddagger}$ Laboratory for Investigative Dermatology, Rockefeller University, New York, New York 10021
}

\begin{abstract}
The skin is constantly exposed to sunlight and frequently develops sun-induced skin cancers such as basal cell carcinoma (BCC). These epidermal-derived tumors escape local immune surveillance and infiltrate the dermis, requiring surgical removal. We report here that in contrast to keratinocytes in normal skin $(n=4)$, BCC tumor cells $(n=6)$ strongly and diffusely express Fas ligand (CD95L), but not Fas antigen (CD95). This CD95L expression in vivo by BCC tumor cells is associated with peritumoral $\mathrm{T}$ lymphocytes that are undergoing apoptosis. Moreover, CD95L can be induced on normal cultured keratinocytes after exposure to ultraviolet-B light (UV-B) irradiation. This induction of CD95L was confirmed at the mRNA and protein levels using multipassaged human keratinocytes and a keratinocyte cell line. Keratinocytes induced to express CD95L acquired the functional capacity to kill a CD95-positive lymphocyte cell line. Whereas hyperplastic keratinocytes in untreated psoriatic plaques do not express CD95L on their plasma membrane, after UV-B treatment there is strong and diffuse keratinocyte CD95L expression that coincided in a temporal fashion with depletion of intraepidermal $T$ cells in all five patients studied. Our data suggest a novel molecular pathway by which UV light can contribute to the ability of a skin cancer to escape from immune attack by cytotoxic $T$ lymphocytes, and a previously unrecognized therapeutic mechanism of action for UV-B light in psoriasis via keratinocyte CD95L expression. Such immunological events involving CD95L provide new insight and opportunity for novel treatment approaches not only for cutaneous neoplasms but also for various $\mathrm{T}$ cell-mediated dermatoses such as psoriasis. (J. Clin. Invest. 1998. 101:33-39.) Key words: skin cancer • keratinocytes $\bullet$ CD95 • CD95L • apoptosis
\end{abstract}

C. Gutierrez-Steil and T. Wrone-Smith contributed equally to this work.

Address correspondence to Dr. Brian J. Nickoloff, Loyola University Medical Center, Skin Cancer Research Laboratories, Cardinal Bernardin Cancer Center-Room 301, 2160 South First Avenue, Maywood, IL 60153. Phone: 708-327-3241; FAX: 708-327-3239; E-mail: bnickol@luc.edu

Received for publication 10 July 1997 and accepted in revised form 17 October 1997.

J. Clin. Invest.

(C) The American Society for Clinical Investigation, Inc. 0021-9738/98/01/0033/07 \$2.00

Volume 101, Number 1, January 1998, 33-39

http://www.jci.org

\section{Introduction}

Basal cell carcinomas (BCCs) $)^{1}$ occur primarily on sun-exposed skin, and are the most common malignancy in humans, with an estimated 750,000 cases per year in the United States (1). The tumor cells in BCC have several different possible methods for evading immune recognition and destruction by cytotoxic $\mathrm{T}$ lymphocytes. Four previously described immunologic aberrations in BCC include (a) absence of cell surface expression of class II MHC antigens (HLA-DR) and intercellular adhesion molecule 1 (CD54) (2); (b) production of IL-10, a cytokine that suppresses expression of costimulatory molecules B7-1 (CD80) and B7-2 (CD86) by professional (i.e., dendritic) antigen-presenting cells, inhibiting their ability to activate $\mathrm{T}$ cells (3-5); (c) overexpression of cell survival gene products such as Bcl-2; and $(d)$ failure of tumor cells to express the Fas antigen (CD95), thereby avoiding T cell killing by Fas ligand (CD95L)bearing cytotoxic $\mathrm{T}$ cells (6-8).

Recently, much attention has focused on the role of CD95 and $\mathrm{CD} 95 \mathrm{~L}$ in the regulation of costimulation and immune responses $(9,10)$. To identify specific molecular events that impact cell-mediated immune reactions in the skin $(11,12)$, we asked whether tumor cells of the most common malignancy in humans aberrantly express CD95L. Such expression would potentially imbue the tumor cell with the potential to kill infiltrating T cells expressing CD95 by inducing apoptosis $(13,14)$. Previously, we observed that $\mathrm{T}$ cells immediately adjacent to BCC were undergoing apoptosis, whereas $\mathrm{T}$ cells not in proximity to tumor cells were viable, indicating that tumor cells could be responsible for induction of programmed cell death in the peritumoral $\mathrm{T}$ cells (8). In addition, we examined the benign hyperplastic keratinocytes in psoriatic plaques before and after exposure to ultraviolet-B (UV-B) light. Such UV-B exposure can induce long-lasting clinical remissions, but the molecular mechanism by which intraepidermal $\mathrm{T}$ cells are depleted has not been elucidated previously (15).

In this report, we document strong and diffuse expression of CD95L by infiltrating tumor cells of BCC. Using cultured multipassaged human keratinocytes and a keratinocyte cell line, induction of CD95L was modulated by cytokines, a tumor promoter, and UV-B light. Using an in vitro cytotoxicity assay, keratinocytes induced to express CD95L acquired the capacity to kill CD95-positive lymphocytes. Furthermore, in vivo exposure of psoriatic skin to UV-B light also led to induction of strong and diffuse CD95L expression by keratinocytes that coincided with depletion of intraepidermal T cells (15). These data provide a molecular basis by which tumor cells can suppress cell-mediated immunity, and new insight into the mechanism of action of UV-B light in producing clinical remissions of psoriasis involving CD95 and CD95L.

1. Abbreviations used in this paper: BCC, basal cell carcinoma; TPA, 12- $O$-tetradecanoyl phorbol 13-acetate. 


\section{Methods}

Human tissue samples. All tissue samples in the study were obtained from biopsies of skin that were immediately snap-frozen and stored at $-80^{\circ} \mathrm{C}$ until analysis. Tissue samples included four different biopsies of normal adult human skin and six different patients with BCC. All tumors were removed from sun-exposed areas of the face. Five patients (three males and two females; age range 32-62 yr) with psoriasis all had extensive and active plaque-stage disease (15\% of body surface or greater). 6-mm punch biopsies of symptomless skin and psoriatic plaques before, during (days 11-15), and after (days 30-34) UV-B treatment were obtained as described previously (15). Briefly, patients were admitted to Rockefeller University for Gockerman treatment in which a $3 \%$ coal tar emollient was used with UV-B exposure. Initial UV-B doses were determined by minimal erythema dose testing on day 1 , with $15 \%$ incremental daily doses as tolerated. The initial UV-B treatment was $\sim 80 \%$ of the minimal erythema dose and ranged between 35 and $70 \mathrm{~mJ} / \mathrm{cm}^{2}$. The mean UV-B dose at midtreatment was $165 \mathrm{~mJ} / \mathrm{cm}^{2}$, and at completion was $608 \mathrm{~mJ} / \mathrm{cm}^{2}$. Skin was irradiated in a unit (model 5700; Psoralite Corp., Columbia, SC) containing LS-FS7T12-UVB-HO fluorescent bulbs, which emit in a broad peak of 285-345 nm except for a spike of increased irradiation at $313 \mathrm{~nm}$. UV-B output was measured with a radiometer (model IL 1700; International Light, Inc., Newburyport, MA) and calibrated UV-B detector (model SED 240, International Light, Inc.).

Cells and cell lines. Multipassaged human keratinocytes were obtained from biopsies of healthy skin as described previously (8). Briefly, keratinocytes were grown in plastic petri dishes (Falcon Labware, Becton Dickinson, Franklin Lakes, NJ) in low calcium, serumfree keratinocyte growth medium (Clonetics Corp, San Diego, CA) and maintained at $37^{\circ} \mathrm{C}$ in a humidified incubator with $5 \% \mathrm{CO}_{2}$. For some experiments, cells were grown in eight-well slide chambers (Lab Tek; Nunc, Inc., Naperville, IL). An immortalized keratinocyte cell line (HaCaT cells) (16) was kindly provided by Professor N.E. Fusenig (German Cancer Research Center, Heidelberg, Germany), and was also propagated in keratinocyte growth medium under identical conditions as described above. Cultured keratinocytes were treated with various agents, including the following: $12-O$-tetradecanoyl phorbol 13-acetate (TPA, 5 nM; Sigma Chemical Co., St. Louis, MO), recombinant human IFN- $\gamma\left(10^{3} \mathrm{U} / \mathrm{ml}\right.$; Genentech Inc., S. San Francisco, CA), recombinant human TNF- $\alpha\left(10^{3} \mathrm{U} / \mathrm{ml}\right.$; Genentech Inc.), and UV-B light. Human colon epithelial cell line SW620 was obtained from American Type Culture Collection (Rockville, MD) and grown in DME plus $10 \%$ FCS as described previously (17). The murine lymphocytic leukemia line L1210.3 (nontransfected) and L1210.Fas (transfected with mouse CD95) and the human chronic myelogenous leukemia cell line K-562-neo.F10 (transfected with vector control) and K-562-hFL.E12 (transfected with human CD95L) were kindly donated by Richard Duke (University of Colorado, Denver, CO). These cell lines were cultured in RPMI medium supplemented with $10 \%$ FCS and $10^{-4} \mathrm{M} \beta$-mercaptoethanol. Transfected cell lines were maintained in $600 \mu \mathrm{g} / \mathrm{ml}$ geneticin (GIBCO BRL, Gaithersburg, MD).

${ }^{51}$ Cr-release assay for CD95/CD95L-mediated cytotoxicity. Cytotoxicity tests were carried out in $\mathrm{V}$-shaped microtiter plates with ${ }^{51} \mathrm{Cr}$ labeled target cells $(5,000$ cells/well) and effector cells at the indicated ratios in a total volume of $100 \mu \mathrm{l} /$ well in RPMI medium with $10 \%$ FCS. The target cells were L1210.3 (CD95-negative) and L1210.Fas (CD95-positive) murine B lymphocytic leukemia cell lines. The effector cells included K562-neo.F10, K562-hFL.E12, SW620 colon epithelial cells, and human keratinocytes untreated or treated with $10^{3} \mathrm{U} / \mathrm{ml}$ IFN- $\gamma$ and $5 \mathrm{nM}$ TPA or $30 \mathrm{~mJ} / \mathrm{cm}^{2}$ UV-B light for $18 \mathrm{~h}$. Human keratinocytes and SW620 cells were treated briefly with $10 \mathrm{mM}$ EDTA and washed before coculture with target cells. Use of EDTA was important to prevent the activation of proteases which can degrade membrane-bound CD95L (18). Plates were centrifuged briefly, and target/effector cells were incubated for $18 \mathrm{~h}$. To address CD95L-specific killing of target cells by SW620 or keratinocytes, Fas.Fc fusion protein (human CD95 fused to Fc portion of mouse $\mathrm{IgG1}$, a kind gift from Gary Nabel, University of Michigan, Ann Arbor, MI; reference 19) was included in some wells. Spontaneous release was determined in wells containing target cells in medium alone, and maximum release was determined in wells containing target cells in medium containing $1.0 \%$ Triton X-100. Supernatants were harvested and counted in a microplate scintillation and luminescence counter (Topcount; Packard, Meriden, CT). The percentage of specific killing was calculated as

$100 \times([$ sample release ] $-[$ spontaneous release ] $) /$ ([maximum release $]-[$ spontaneous release $])$.

Immunohistochemical staining. Cryostat sections of skin $(5 \mu \mathrm{m}$ thick) were fixed using acetone and immunostained using an avidinbiotin detection system (Vectastain; Vector Laboratories, Burlingame, CA) as described previously (2). The antibodies were used at optimal concentrations with 3-amino-9-ethylcarbazole (producing a positive red reaction product) as the chromagen with $1 \%$ hematoxylin as the counterstain. Mouse mAb against CD95 (CH11, IgM; Upstate Biotechnology, Inc., Lake Placid, NY) was used, as were two different rabbit anti-human CD95L antisera (Q20 and C20; Santa Cruz Biotechnology, Inc., Santa Cruz, CA). Rhodamine-conjugated mouse $\mathrm{mAb}$ against human $\mathrm{CD} 3$ was purchased from Becton Dickinson (Mountain View, CA).

Western blot analysis. Soluble fractions of cultured keratinocytes of BCC tumor fragments were prepared as described previously (8), except 3-[(3-cholamidopropyl)dimethylammonio]-1-propanesulfonic acid lysis buffer (20) was used instead of Triton buffer. $50 \mu \mathrm{g}$ of each protein sample was electrophoresed under reducing conditions through a $12.5 \%$ SDS-polyacrylamide gel (SDS-PAGE), and transferred to a nitrocellulose membrane (Schleicher \& Schuell, Keene, $\mathrm{NH}$ ). Staining for CD95L was performed using a rabbit polyclonal CD95L antiserum followed by goat anti-rabbit IgG-peroxidase (Jackson ImmunoResearch Labs, West Grove, PA). Protein expression was detected by a chemiluminescent detection system (ECL kit; Amersham Corp., Arlington Heights, IL).

Reverse transcription and PCR. Total cellular RNA was isolated using Trizol reagent (GIBCO BRL), and converted to cDNA using the superscript preamplification system for first-strand cDNA synthesis (GIBCO BRL), with oligo $(\mathrm{dt})_{12-18}$ primer. PCR was performed using the following sense and antisense primers, respectively: Fas receptor, CAGAACTTGGAAGGCCTGCATC and TCTGTTCTGCTGTGTCTTGGAC (17), Fas ligand, CACCCCAGTCCACCCCCTGA and AGGGGCAGGTTGTTGCAAGA (21). $1 \mu 1$ of cDNA was amplified by PCR in a $50-\mu l$ reaction volume containing PCR buffer (20 mM Tris- $\mathrm{HCl}$ [pH 8.4], $50 \mathrm{mM} \mathrm{KCl}, 1.5 \mathrm{mM} \mathrm{MgCl}_{2}, 0.2 \mathrm{mM}$ dNTP, 5 pmol of each primer, and $2.5 \mathrm{U}$ Taq DNA polymerase [GIBCO BRL]). PCR was performed in a GeneAmp PCR system 2400 thermal cycler (Perkin-Elmer Corp., Branchburg, NJ) with the following thermal profile: $94^{\circ} \mathrm{C}$ for 3 min followed by 35 cycles of denaturation at $94^{\circ} \mathrm{C}$ for $30 \mathrm{~s}$, annealing at $55^{\circ} \mathrm{C}$ for $30 \mathrm{~s}$, and extension at $72^{\circ} \mathrm{C}$ for $45 \mathrm{~s}$, and a 10 -min incubation at $72^{\circ} \mathrm{C}$. Products were separated by electrophoresis through a $1.5 \%$ agarose gel and visualized by ethidium bromide staining and UV illumination.

\section{Results}

Distribution of CD95L and CD95 in normal human skin and $B C C$. Even though CD95L was observed initially on activated $\mathrm{T}$ lymphocytes (22), it can also be expressed by several other cell types, including corneal epithelial cells (23). Since the skin and eye share some immunological features (24) and are both exposed continually to UV containing solar rays, we asked if a sunlight-induced tumor of the skin expressed CD95L. We focused initially on BCCs because they are the most frequent tumor in humans and are associated with chronic sunlight exposure (1). We postulated that if the constitutive expression of 


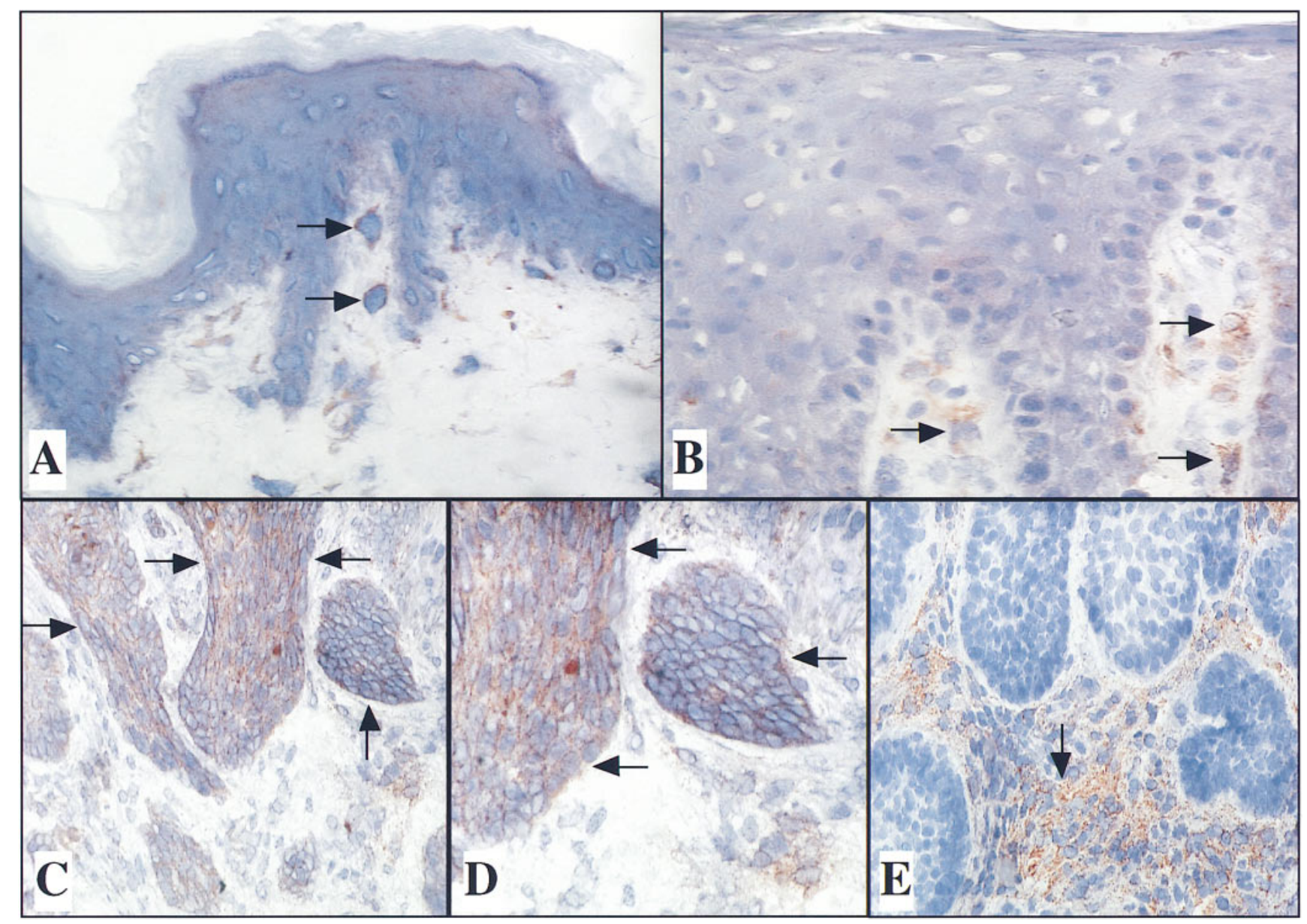

Figure 1. Strong expression of CD95L in BCC but not normal or psoriatic skin. (A) Immunostaining reveals low levels of CD95L expression by keratinocytes in normal adult human skin, although dermal lymphoid cells are positive (arrows). (B) Untreated psoriatic plaque has occasional infiltrating CD95L-positive mononuclear cells (arrows), but minimal keratinocyte CD95L expression. (C) Infiltrating BCC tumor cells strongly and diffusely express CD95L (arrows). (D) Tumor cells in both the center and periphery of islands are CD95L-positive (arrows), as are surrounding mononuclear cells. (E) BCC tumor cells are devoid of CD95, although peritumor stroma has many CD95-positive cells (arrow).

CD95L by the cornea was related to sunlight exposure, then UV irradiation might be one mechanism by which epithelial cells in the skin regulate CD95L expression. Immunohistochemical staining of normal adult skin with either of two distinct rabbit antibodies (designated C20 and Q20) against CD95L revealed absent to low amounts on the plasma membrane of epidermal keratinocytes (Fig. $1 A$ ), consistent with an earlier report that used a different antibody (25). Occasional mononuclear cells in the dermis resembling lymphocytes stained positive for CD95L (Fig. $1 \mathrm{~A}$, arrows), which served as a positive internal control. The staining in this series of patients was judged to be CD95L-specific by several criteria, including (a) use of normal rabbit serum at the same dilution produced no consistent staining of any cells in epidermis or dermis; $(b)$ intensity of the signal diminished as the dilution was increased; $(c)$ studies using Jurkat $\mathrm{T}$ cells revealed the epitope was induced rapidly on the surface after activation; $(d)$ preincubating the anti-CD95L antibody with a blocking antibody abrogated the positive staining; and $(e)$ as portrayed below, use of one of the antibodies (Q20) in Western blot analysis immunoprecipitated the appropriate size molecular species of CD95L. In general, the extents of keratinocyte plasma membrane detection using the C20 and
Q20 antibodies were comparable, but the C20 antibody tended to decorate the cytoplasm of keratinocytes to a greater extent than the Q20 antibody.

When untreated psoriatic plaques were examined, CD95L was not conspicuous on the surface of the hyperplastic keratinocytes, but was present on dermal mononuclear cells, including macrophage and lymphoid-type cells (Fig. $1 B$ ). In normal skin and psoriatic plaques, no CD95L was seen consistently on Langerhans cells or melanocytes, and $<10 \%$ of all epidermal and dermal mononuclear cells in the psoriatic plaques were CD95L-positive (Fig. 1, $A$ and $B$ ). In contrast, tumor cells in all BCCs had invasive islands that were strongly and diffusely positive for CD95L on their plasma membranes (Fig. 1, $C$ and $D)$. Individual cells in the center and at the periphery of the collection of tumors were intensely CD95L-positive. CD95L was also easily detectable on peritumoral mononuclear cells such as macrophages and lymphocytes but not endothelial cells. It should be noted that we observed previously that T lymphocytes immediately surrounding BCC tumor cells were found to be undergoing apoptosis (8). Serial sections of the BCCs revealed complete absence of CD95 expression by the tumor cells, although the surrounding stroma contained innumerable posi- 
tive cells (Fig. $1 E$ ). The absence of CD95 expression by the tumor cells supports the notion that the tumor cells are producing CD95L and not just binding soluble CD95L onto their surface. To demonstrate further that the immunostaining results represented recognition of CD95L, tumor cell fragments were dissected, and the protein extract was subjected to Western blot analysis, which confirmed the presence of a 40-kd molecular species of CD95L (data not shown). Thus, BCC should be added to the rapidly growing list of nonhematolymphoid human malignancies in which tumor cells express CD95L, such as colon carcinoma, melanoma, and hepatocellular carcinoma $(17,23,25,26)$.

Induction of CD95L on cultured human keratinocytes. While it is becoming clear that multiple tumor cell types can express CD95L, little is known regarding the trigger factors that are responsible for the induction of CD95L. Because the skin has been found to be an excellent organ for advancing our understanding of carcinogenesis (27), we next asked how different stimuli implicated in various tumor models would influence cultured keratinocyte CD95L expression. Stimuli selected for these experiments included UV-B irradiation, tumor promoter TPA, and primary cytokines implicated in skin pathology, TNF- $\alpha$ and IFN- $\gamma$. By immunohistochemical staining, untreated multipassaged keratinocytes demonstrate weak cytoplasmic and plasma membrane expression of CD95L (Fig. $2 A$ ). Exposing cultured keratinocytes for $48 \mathrm{~h}$ to TPA (Fig. $2 B$ ), IFN- $\gamma$ (Fig. $2 C$ ), and TNF- $\alpha$ (Fig. 2 D) increased cytoplasmic and plasma membrane expression of CD95L. Combining TPA and IFN- $\gamma$ (Fig. $2 \mathrm{E}$ ) also led to induction of CD95L expres- sion by the keratinocytes. Exposing keratinocytes to $30 \mathrm{~mJ} / \mathrm{cm}^{2}$ UV-B light alone (Fig. $2 F$ ) stimulated CD95L expression in the cytoplasm and on the plasma membrane, producing a chicken-wire pattern of expression resembling the BCC tumor samples. Similar results were observed using either multipassaged keratinocytes obtained from normal human skin or an immortalized keratinocyte cell line, HaCaT (16). No induction of CD95L was observed with keratinocytes exposed to only 10 $\mathrm{mJ} / \mathrm{cm}^{2}$ of UV-B light, and no further increase in CD $95 \mathrm{~L} \mathrm{ex-}$ pression was detectable above $30 \mathrm{~mJ} / \mathrm{cm}^{2}$ (i.e., $50 \mathrm{~mJ} / \mathrm{cm}^{2}$ ). Interestingly, while there was constitutive expression of CD95 by cultured keratinocytes, exposure to UV-B light abrogated completely any detectable CD95 expression by immunostaining (data not shown). Thus, UV-B light treatment of cultured keratinocytes produced a phenotype (CD95-negative, CD95Lpositive) resembling BCC. Because CD95L is sensitive to proteases such as trypsin (required to prepare single-cell suspensions), we could not use flow cytometry on cultured keratinocytes to quantify CD95L expression. Using Western blot analysis, the immunohistochemical staining results were extended, and untreated keratinocytes displayed only faint bands at 40 and $27 \mathrm{kD}$ (Fig. $3 \mathrm{~A}$ ). However, treatment of cultured keratinocytes with UV-B light (Fig. 3, lanes $C$ and $E$ ) or IFN- $\gamma$ plus TPA (Fig. 3, lanes $B$ and $D$ ) increased expression at 24 and $48 \mathrm{~h}$ for the 27- and 40-kD forms of CD95L. These molecular species of CD95L have been observed previously for other (i.e., nonkeratinocyte) cell types $(25,26)$. The increase in the $40-\mathrm{kD}$ CD95L protein molecule over the untreated control keratinocytes was fourfold for IFN- $\gamma$ plus TPA, and fourfold for

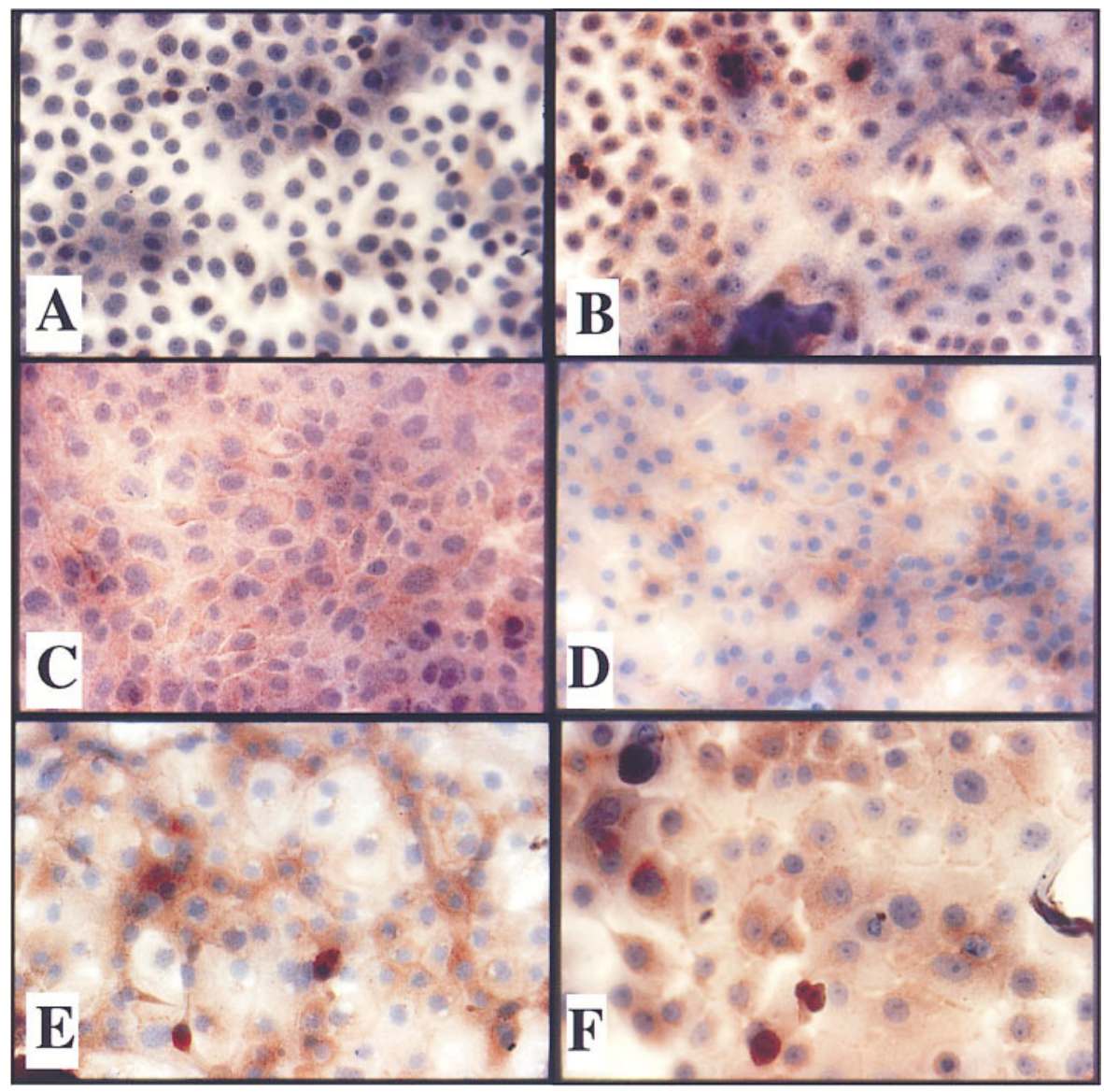

Figure 2. Induction of $\mathrm{CD} 95 \mathrm{~L}$ on cultured human keratinocytes. $(A)$ Without stimulation, keratinocytes express low amount of CD95L. Increased cytoplasmic and plasma membrane CD95L expression of keratinocytes exposed to TPA $(B), \operatorname{IFN}-\gamma(C)$, TNF- $\alpha(D)$, IFN- $\gamma$ plus TPA $(E)$, and after UV-B light treatment $\left(30 \mathrm{~mJ} / \mathrm{cm}^{2}\right)(F)$. 


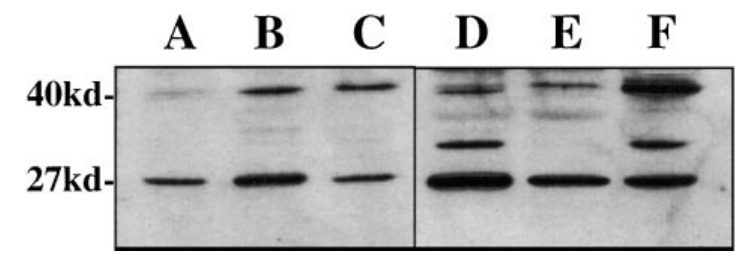

Figure 3. Induction of CD95L protein by cultured keratinocytes. Immunoblot analysis reveals that untreated keratinocytes (lane $A$ ) have low constitutive amounts of the 40-kD species of CD95L, but more of the 27-kD species. After exposure to IFN- $\gamma$ plus TPA for 24 and $48 \mathrm{~h}$ (lanes $B$ and $D$ ), or UV-B light $\left(30 \mathrm{~mJ} / \mathrm{cm}^{2}\right)$ for 24 and $48 \mathrm{~h}$ (lanes $C$ and $E$ ), respectively, there was an increase in the amount of the $40 \mathrm{kD}$ species of CD95L. For comparison, protein extraction from SW620 cells was also performed (lane $F$ ). The nonglycosylated form of CD95L can be seen in lanes $D$ and $F(32 \mathrm{kD})$.

UV-B light. The 32-kD CD95L protein band (Fig. 3, lanes $D$ and $F$ ) represents the nonglycosylated form as described previously (18). To confirm further that cultured keratinocytes could indeed produce CD95L, reverse transcription-PCR analysis was performed. Using previously published primer sets for CD95L mRNA, cultured keratinocytes were found to be positive for CD95L mRNA (data not shown).

$U V$-B light-exposed keratinocytes acquire ability to induce T lymphocyte cytotoxicity. As observed previously, it is rare for viable $\mathrm{T}$ lymphocytes to be seen binding to or contained within the islands of tumor cells in biopsies of BCC (2). Given the striking expression of CD95L by BCC, and the upregulation of CD95L by cultured keratinocytes, we next asked if UV-B light-treated keratinocyte cultures acquired the capacity to kill a Fas-expressing lymphoma cell line. The cytoxicity assays were conducted with two different target cell types, a murine lymphoma line expressing no CD95, and the same line transfected with CD95 (detectable by flow cytometry; data not shown). The functional activity of CD95L expressed by the keratinocytes revealed (Fig. 4, left) that whereas untreated keratinocytes induced $<10 \%$ of the specific killing of CD95-positive lymphoma cells, both IFN- $\gamma$ plus TPA-treated keratinocytes and UV-B light-exposed keratinocytes acquired the capacity to induce two- to threefold higher levels of specific killing, reaching $30 \%$ for the UV-B light-exposed keratinocytes $(P<$ $0.01)$. This cytoxicity was inhibited almost completely by a Fas.Fc fusion protein, indicating that lysis was due to CD95CD95L interaction. Furthermore, there was no significant lysis of lymphocytes not expressing CD95. The UV-B light-exposed keratinocytes were either equal or better at inducing specific killing of the CD95-positive target cells compared with CD95Lexpressing SW620 cells or K562 cells transfected with CD95L, or when the target cells were exposed to an anti-CD95 antibody (Fig. 4, right).

Successful treatment of psoriasis using $U V$-B light induces keratinocyte $C D 95 \mathrm{~L}$. To examine the human skin response in vivo, five patients with active untreated psoriatic plaques were studied before, during, and after induction of clinical remission using UV-B light therapy as described previously (Fig. 5) (15). Untreated psoriatic skin was negative for CD95L expression on the plasma membrane of the hyperplastic keratinocytes in all patients (Fig. $5 \mathrm{~A}$ ). In two patients, scattered epidermal keratinocytes in the clinically symptomless skin had focal CD95L expression. In biopsies of skin taken 2-3 wk after initiation of
UV-B light therapy, there was increased CD95L expression by epidermal keratinocytes in all skin samples of both psoriatic plaques (Fig. 5 B) and symptomless skin (Fig. 5 E). This marked enhancement of CD95L expression was also observed on epidermal keratinocytes after the completion (4-5 wk) of UV-B light therapy (Fig. 5, $C$ and $F$ ). It should be noted that the temporal appearance of CD95L by keratinocytes in the UV-B light-treated psoriatic plaques coincided with the disappearance of T cells (15).

While there was $51 \pm 8$ intraepidermal CD3 + T cells present in the pretreatment psoriatic plaque biopsies, after $2-3 \mathrm{wk}$ of UV-B light treatment, only $9 \pm 3$ intraepidermal T cells were present in the skin biopsies. There was no light microscopic evidence of any cytopathic effects of UV-B light treatment on the keratinocytes, as their nuclei had evenly dispersed chromatin with no fragmentation or clumping.

\section{Discussion}

Chronic and near-continuous exposure to solar UV irradiation are hallmarks for both ocular and cutaneous organ systems. Neither the skin nor the eyes can tolerate destructive inflammatory reactions or chronic immune-mediated processes (23, 24). Since CD95-CD95L interactions have been observed pre-

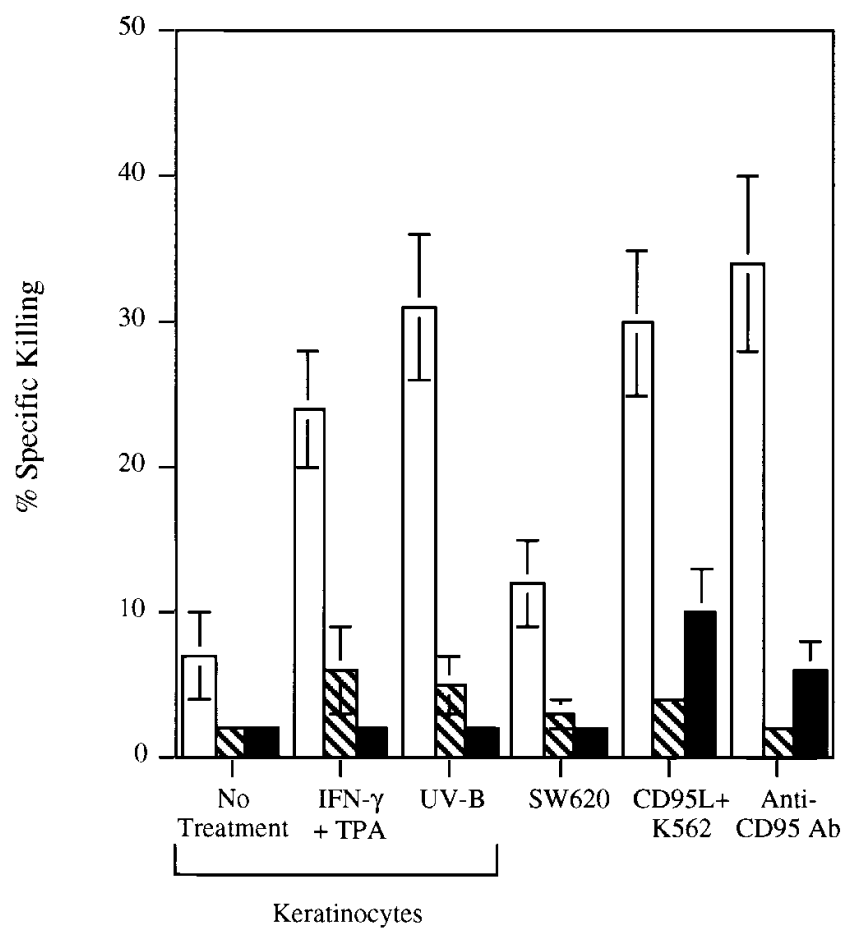

Figure 4. CD95L-positive keratinocytes kill CD95-positive lymphocytes. Cytoxicity assays (\% Specific Killing) reveal that IFN- $\gamma$ plus TPA-treated or UV-B light-exposed keratinocytes induce specific killing of CD95-positive lymphocytes greater than or equal to killing mediated by SW620 cells, K562 cells transfected with CD95L, or antiCD95 antibody. Effector to target ratio was 5:1, with targets including either CD95-positive (white bars) or CD95-negative (hatched bars) lymphocytes. Black bars, CD95-positive lymphocytes incubated with a Fas.Fc fusion protein. For all experiments, target and effector cells were coincubated for $18 \mathrm{~h}$, and ${ }^{51} \mathrm{Cr}$ release was measured as described in Methods. Results shown represent mean \pm SD for four independent experiments. 


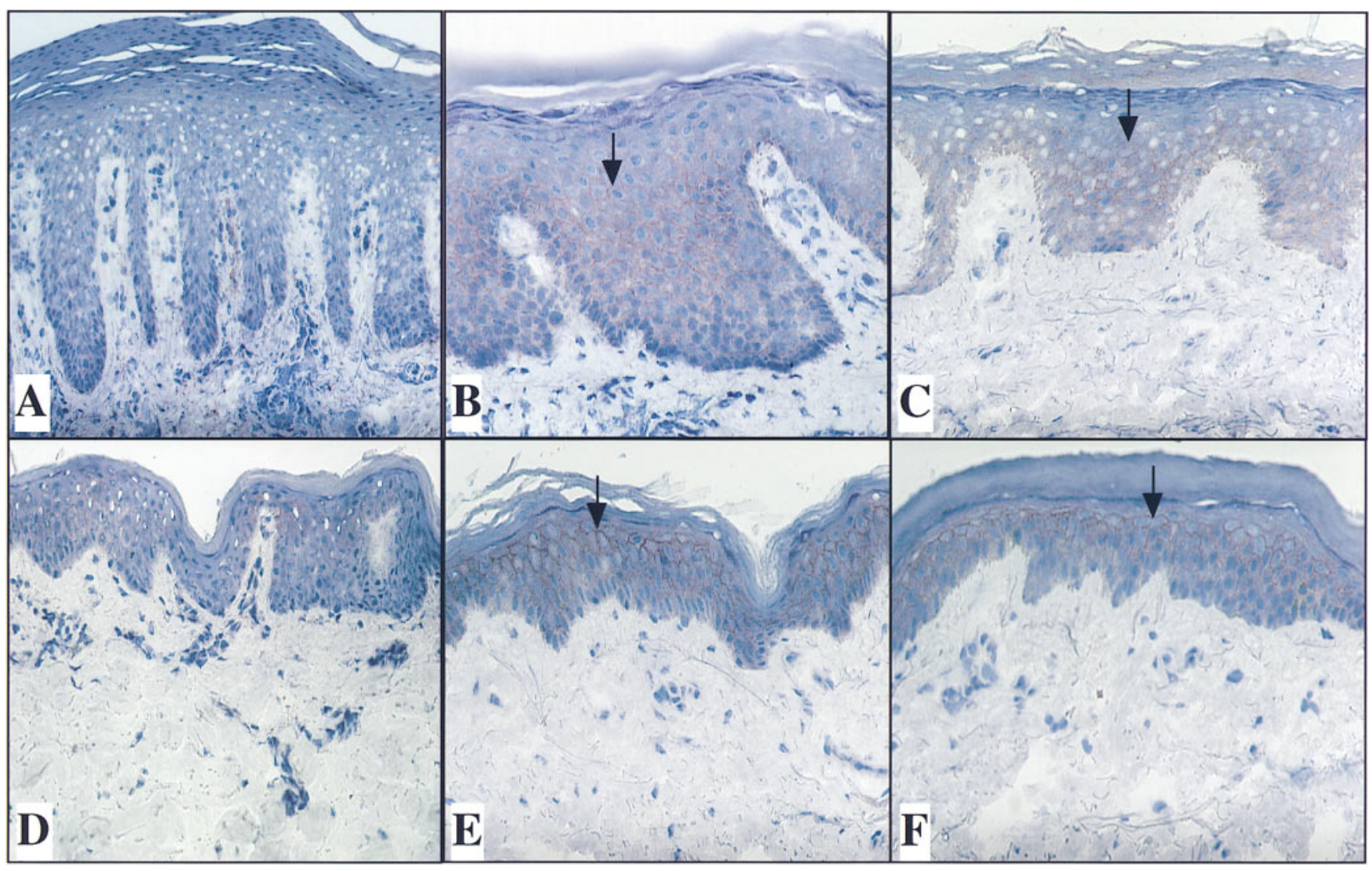

Figure 5. Irradiation of skin by UV-B light induces keratinocyte CD95L expression. Both psoriatic plaques $(A-C)$ and adjacent symptomless skin $(D-F)$ from the same patient were immunostained to detect CD95L. Before treatment $(A$ and $D)$, low levels of CD95L were detectable on epidermal keratinocytes. $2(B$ and $E)$ and $4 \mathrm{wk}(C$ and $F)$ after initiation of daily UV-B light exposure, keratinocytes are induced to express $\mathrm{CD} 95 \mathrm{~L}$ on their plasma membranes. Note the decreased thickness of the epidermis and diminution in number of mononuclear cells in the psoriatic lesion after UV-B light treatment (compare $A$ with $B$ and $C$ ).

viously to play important roles in modulating inflammatory and immunological reactions in other organ systems, we explored the potential relationship between UV irradiation and keratinocytes with regard to these cell surface molecules. The current results provide some insight, establishing for the first time a direct link between UV-B light exposure and epithelial cell CD95L expression. On the one hand, corneal epithelial expression of CD95L is beneficial in maintaining immune privilege such that inflammatory and immune-mediated $\mathrm{T}$ cell assaults in the eye are kept to a minimum (24). However, it appears that tumor cells in the skin can use the UV-B lightmediated induction of CD95L to escape from a local immune response that includes infiltrating cytotoxic T lymphocytes (3, 27 ). In our previous report (8), we emphasized that $\mathrm{T}$ cells immediately adjacent to islands of BCC tumor were frequently TUNEL-positive (for terminal deoxynucleotidyl transferasemediated dUTP-biotin nick end-labeling, a marker for cells undergoing apoptosis), whereas $\mathrm{T}$ cells not in proximity to tumor cells did not undergo apoptosis. The striking and diffuse expression of CD95L by the tumor cells provides a molecular basis for these tissue patterns of apoptotic T cells. It will be of interest to determine if melanocytes/melanoma cells will respond to UV-B light in the same way as keratinocytes, by upregulating CD95L and suppressing CD95 expression (25). Preliminary studies reveal that the other sunlight-induced ma- lignancy of skin, squamous cell carcinoma, also has infiltrating tumor cells positive for CD95L expression. The tumor cells in BCC appear to have created an optimal regional immunosuppressive microenvironment, in that the tumor cells fail to express HLA-DR, intercellular adhesion molecule 1, leukocyte function-associated antigen 3, and CD95, and at the same time produce IL-10 and CD95L. Such a coordinated and highly effective series of molecular events that are widely and uniformly dispersed among infiltrating islands of tumor cells suggests a proximal controlling event under the influence of a UV-B light-sensitive regulatory element(s), which merits further attention.

Not only can UV-B light have a direct influence on keratinocyte CD95L expression, but a tumor promoter (i.e., TPA) and primary cytokines produced by keratinocytes and the skin immune system in response to sunlight exposure (i.e., TNF- $\alpha$ and IFN- $\gamma$ ) (28) can also stimulate CD95L expression. To our knowledge, this is the first demonstration that such stimuli (in addition to UV-B light) can modulate CD95L expression in an epithelial cell type. This induction of CD95L by keratinocytes imbued these cells (particularly the UV-B light-exposed keratinocytes) with the functional ability to mediate specific killing of lymphocytes expressing CD95.

Besides these in vitro results, we examined the in vivo response in psoriatic patients with respect to UV-B light and ke- 
ratinocyte $\mathrm{CD} 95 \mathrm{~L}$ expression. We observed that in untreated active psoriatic lesions, hyperplastic keratinocytes do not express any CD95L on their plasma membranes, but after UV-B light treatment, lesional keratinocytes strongly express CD95L, with a chicken-wire appearance. Such induction of CD95L by UV-B light may explain how this light treatment can improve the psoriatic plaques by mediating apoptosis of infiltrating pathogenic epidermal T cells. A previous clinical trial demonstrated clearly that UV-B light treatment of psoriatic plaques was associated with selective depletion of intraepidermal $\mathrm{T}$ lymphocytes (15). Because of the strong and diffuse positive labeling in psoriatic epidermis by terminal deoxynucleotidyl transferase-mediated dUTP-biotin nick end-labeling assay, we could not reliably use this technique to assess apoptosis before or after UV-B light treatment (29). However, based on the light microscopic appearance, the keratinocytes displayed no morphological evidence of any cytopathic changes at midpoint (2-3 wk) or completion (4-5 wk) of UV-B light treatment. Studies are under way using a severe combined immunodeficient mouse model of psoriasis (30) to determine if preirradiation of symptomless skin engrafted onto severe combined immunodeficient mice can suppress the ability of injected pathogenic $\mathrm{T}$ cells to produce full-fledged psoriatic plaques. Furthermore, it will be interesting to determine if an abnormality in keratinocyte CD95L induction by UV-B light and subsequent interaction with CD95-positive T cells is relevant to patients with SLE (31).

In conclusion, keratinocytes can be induced to express functionally active CD95L, and, thus, yet another immunomodulatory function of these epithelial cells can be appreciated (i.e., potential terminators of immune reactions by killing infiltrating CD95-positive T cells) beyond those already recognized for keratinocytes (32). Additional clinical studies are necessary to determine if negating CD95L expression by tumor cells in BCC can have a positive therapeutic outcome.

\section{Acknowledgments}

The expert technical assistance of Jeffrey Panella, Patricia Bacon, and Dorothy Fedor is gratefully acknowledged. We are indebted to Dr. Jeffrey Melton and Jason Swanson for tissue procurement, and to Drs. Richard Duke and Gary Nabel for cell lines and reagents.

This work was partially supported by National Institutes of Health grants AR-38957, 40065, and 40488 (to B.J. Nickoloff), and RR-00102 and AI-39214 (to J.G. Krueger).

\section{References}

1. Miller, D.L., and M.A. Weinstock. 1994. Nonmelanoma skin cancer in the United States: incidence. J. Am. Acad. Dermatol. 30:774-778.

2. Taylor, R.S, C.E.M. Griffiths, M. Brown, N. Swanson, and B.J. Nickoloff. 1990. Constitutive absence and interferon-gamma induction of expression of adhesion molecules in basal cell carcinoma. J. Am. Acad. Dermatol. 22:721-726.

3. Kim, J., R.L. Modlin, R.L. Moy, S.M. Dubinett, T. McHugh, B.J. Nickoloff, and K. Uyemura. 1995. IL-10 production in cutaneous basal and squamous cell carcinoma. A mechanism for evading the local $\mathrm{T}$ cell immune response. J. Immunol. 155:2240-2247.

4. Mitra, R.S., L.A. Turka, T.A. Judge, F.O. Nestle, and B.J. Nickoloff. 1995. Psoriatic skin-derived dendritic cell function is inhibited by exogenous IL-10. Differential modulation of B7-1 (CD80) and B7-2 (CD86) expression. J. Immunol. 154:2668-2677.

5. Nestle, F.O., G. Burg, J. Fah, T. Wrone-Smith, and B.J. Nickoloff. 1997. Human sunlight-induced basal cell carcinoma are deficient in T cell co-stimulatory molecules and are impaired as antigen-presenting cells. Am. J. Pathol. 150:
$1-11$.

6. Nakagawa, K., K. Yamamura, S. Maeda, and M. Ichihashi. 1994. Bcl-2 expression in epidermal keratinocyte diseases. Cancer. 74:1720-1724.

7. Oishi, M., K. Maeda, and S. Sugiyama. 1994. Distribution of apoptosismediating Fas antigen in human skin and effects of anti-Fas monoclonal antibody on human epidermal keratinocytes and squamous carcinoma cell lines. Arch. Dermatol. Res. 286:396-407.

8. Wrone-Smith, T., G. Nunez, T. Johnson, B. Nelson, L.H. Boise, C.B. Thompson, and B.J. Nickoloff. 1995. Discordant expression of Bcl-x and Bcl-2 by keratinocytes in vitro and psoriatic keratinocytes in vivo. Am. J. Pathol. 146:1-10.

9. Lynch, D.H., F. Ramsdell, and M.R. Alderson. 1995. Fas and FasL in the homeostatic regulation of immune responses. Immunol. Today. 16:569-574.

10. Van Parijs, L., A. Ibraghimov, and A.K. Abbas. 1996. The roles of costimulation and Fas in $\mathrm{T}$ cell apoptosis and peripheral tolerance. Immunity. 4: 321-328.

11. Nickoloff, B.J., R.S. Mitra, J. Green, C. Thompson, L. Turka, and Y. Shimizu. 1993. Discordant expression of CD28 ligands, BB-1, and B7 on keratinocytes in vitro and psoriatic cells in vivo. Am. J. Pathol. 142:1029-1040.

12. Nickoloff, B.J., and L.A. Turka. 1994. Immunological functions of nonprofessional antigen-presenting cells: new insights from studies of T-cell interactions with keratinocytes. Immunol. Today. 15:464-469.

13. Yonehara, S., A. Ishii, and M. Yonehara. 1989. A cell-killing monoclonal antibody (anti-Fas) to a cell surface antigen co-downregulated with the receptor of tumor necrosis factor. J. Exp. Med. 169:1747-1756.

14. Trauth, B.C., C. Klas, A.M. Peters, S. Matzku, P. Moller, W. Falk, K.M Debatin, and P.H. Kramer. 1989. Monoclonal antibody-mediated tumor regression by induction of apoptosis. Science. 245:301-305.

15. Krueger, J.G., J.T. Wolfe, R.T. Nabeya, V.P. Vallat, P. Gilleaudeau, N.S. Heftler, L.M. Austin, and A.B. Gottlieb. 1995. Successful ultraviolet B treatment of psoriasis is accompanied by a reversal of keratinocyte pathology and by selective depletion of intraepidermal T cells. J. Exp. Med. 182:20572068

16. Boukamp, P., R.T. Petrussevska, D. Breitcreutz, J. Hornung, A. Marhan, and N.E. Fusenig. 1988. Normal keratinization in a spontaneously immortalized aneuploid human keratinocyte cell line. J. Cell. Biol. 106:761-777.

17. O'Connell, J., G.C. O'Sullivan, J.K. Collins, and F. Shanahan. 1996. The Fas counterattack: Fas-mediated $\mathrm{T}$ cell killing by colon cancer cells expressing Fas ligand. J. Exp. Med. 184:1075-1082.

18. Mariani, S.M., B. Matiba, C. Baumler, and P.H. Krammer. 1995. Regulation of cell surface APO-1/Fas (CD95) ligand expression by metalloproteases. Eur. J. Immunol. 25:2303-2307.

19. Arai, H., S.Y. Chan, D.K. Bishop, and G.J. Nabel. 1997. Inhibition of the alloantibody response by CD95 ligand. Nat. Med. 3:843-848.

20. Liles, W.C., J.A. Ledbetter, A.W. Waltersdorph, and S.J. Klebanoff. 1995. Crosslinking of CD45 enhances activation of the respiratory burst in response to specific stimuli in human phagocytes. J. Immunol. 155:2175-2184.

21. Oshimi, Y., S. Oda, Y. Honda, S. Nagata, and S. Miyayaki. 1996. Involvement of Fas ligand and Fas-mediated pathway in the cytotoxicity of human natural killer cells. J. Immunol. 157:2909-2915.

22. Suda, T., T. Takahashi, P. Golstein, and S. Nagata. 1993. Molecular cloning and expression of the Fas ligand, a novel member of the tumor necrosis factor family. Cell. 75:1169-1178.

23. Griffith, T.S., T. Brunner, S.M. Fletcher, D.R. Green, and T.A. Ferguson. 1995. Fas ligand-induced apoptosis as a mechanism of immune privilege. Science. 270:1189-1192.

24. Zierhut, M., T. Bieber, E.B. Brocker, J.U. Forrester, C.S. Foster, and J.W. Streilein. 1996. Immunology of the skin and the eye. Immunol. Today. 17: 448-450.

25. Hahne, M., D. Rimoldi, M. Schroter, P. Romero, M. Schreier, L.E. French, P. Schneider, T. Bornard, A. Fontana, D. Lienard, et al. 1996. Melanoma cell expression of Fas (Apo-1/CD95) ligand: implications for tumor immune escape. Science. 274:1363-1366.

26. Strand, S., W.J. Hofmann, H. Hug, M. Muller, G. Otto, D. Strand, S.M. Mariani, W. Stremmel, P.H. Krammer, and P.R. Galle. 1996. Lymphocyte apoptosis induced by CD95 (Apo-1/Fas) ligand-expressing tumor cells. A mechanism of immune evasion? Nat. Med. 2:1361-1366.

27. Yuspa, S.H. 1994. The pathogenesis of squamous cell cancer: lessons learned from studies of skin carcinogenesis-thirty-third G.H.A. Clowes Memorial Award Lecture. Cancer Res. 54:1178-1189.

28. Barker, J.N., R.S. Mitra, C.E.M. Griffiths, V.M. Dixit, and B.J. Nickoloff. 1991. Keratinocytes as initiators of inflammation. Lancet. 337:211-214.

29. Wrone-Smith, T., R.S. Mitra, C.B. Thompson, R. Jasty, V.P. Castle, and B.J. Nickoloff. 1997. Keratinocytes derived from psoriatic plaques are resistant to apoptosis compared with normal skin. Am. J. Pathol. 151:1321-1329.

30. Wrone-Smith, T., and B.J. Nickoloff. 1996. Dermal injection of immunocytes induces psoriasis. J. Clin. Invest. 98:1879-1887.

31. Nagata, S., and T. Suda. 1995. Fas and Fas ligand: lpr and gld mutations. Immunol. Today. 16:39-43.

32. Nickoloff, B.J., and L.A. Turka. 1993. Keratinocytes: key immunocytes of the integument. Am. J. Pathol. 143:325-331. 